\title{
NOTA TECNICA
}

\section{EVALUACION DE LA RESISTENCIA A LA ROYA (Uromyces appendiculatus) EN 64 VARIEDADES DE FRIJOL COMUN EN CUBA ${ }^{1}$}

\author{
Miguel González ${ }^{2}$, Evelio García ${ }^{3}$
}

\begin{abstract}
RESUMEN
Evaluación de la resistencia a la roya (Uromyces appendiculatus) en 64 variedades de frijol común en Cuba. Se evaluaron 64 variedades de frijol común (Phaseolus vulgaris L.) frente al ataque de la roya en la Subestación de Granos de Velasco, provincia de Holguín, Cuba. La evaluación del grado de ataque se efectuó a los 60 días de la siembra, tanto en porcentaje a través de una escala de grados como mediante el conteo de pústulas por hoja. No hubo diferencias estadísticas entre ambos métodos. Además se determinó el grado de susceptibilidad de las variedades, a través de la medición del diámetro de las pústulas considerando tres categorías: grandes (mayor de $0,5 \mathrm{~mm}$ ), medianas (entre $0,3-0,5 \mathrm{~mm}$ ) y pequeñas (menor de $0,3 \mathrm{~mm}$ ) y la presencia o no de halo clorótico en las mismas. Los cultivares de mejor comportamiento ante la enfermedad fueron Güira-35, XAN-43, Revolución-79, M-112, Rosas, BAT-202, Tazumal, Huetar, Bolita-42, BAT-1281, BAT-1274, BAT-1280, PV555, BAT 1275, Engañador, Guamá-23, MCD-254, Chévere, XAN-93, XAN-147, NAG-20, NAG-55, RAB-30 y CC 25-9-S (R) con un promedio igual o inferior a 10 pústulas por hoja, en general pequeñas y sin clorosis.
\end{abstract}

\begin{abstract}
Evaluation of the resistance of 64 common beans (Uromyces appendiculatus) varieties to the rust in Cuba. The varieties (64) of common beans (Phaseolus vulgaris L.) were evaluated for resistance to bean rust in the Velasco zone, at the north of the Holguín province, Cuba. The evaluation was made 60 days after sowing by determining the degree of attack using a scale as well as estimating the number of pu stules per leaf and no remarkable differences between both methods were found. The character of the pustules was also determined by measuring their diameters with regard to three categories large (more than $0.5 \mathrm{~mm}$ ) middling (between 0.3 and $0.5 \mathrm{~mm}$ ) and small (less than $0.3 \mathrm{~mm}$ ) and the presence or lack of chlorotic halo surrounding the pustules as well. The cultivars that resulted less attacked by the bean rust were Guira-35, XAN-43, Revolución-79, M-112, Rosas, BAT-202, Tazumal, Huetar, Bolita-42, BAT-1281, BAT-1274, BAT-1280, PV-555, BAT 1275, Engañador, Guamá23, MCD-254, Chevere, XAN-93, XAN-147, NAG-20, NAG-55, RAB-30 and CC 25-9-S (R), all of which showed an average equal or inferior to 10 pustules per leaf, being these ones generally smaller and without any chlorosis.
\end{abstract}

\section{INTRODUCCION}

La roya (Uromyces appendiculatus) es la enfermedad fúngica más importante en el cultivo del frijol en Cuba, no sólo por estar distribuída en todo el territorio nacional sino por las pérdidas que ocasiona en las cosechas (González, 1984).

En la zona de Velasco, la mayor productora de frijol en Cuba, ubicada en la región Nororiental del país, la roya se ha incrementado en los últimos años y esta circunstancia podría ser atribuída a la siembra reiterada y casi exclusiva de la variedad ICA- Pijao (Gálve; Orozco; Voysest, 1985).
Los objetivos del presente trabajo fueron conocer el grado de resistencia a la roya de las variedades de frijol con potencial de uso comercial.

\section{MATERIALES Y METODOS}

\section{Primer experimento}

En la Subestación de Granos de Velasco, provincia de Holguín, se realizó un experimento con un grupo de 34 variedades de frijol dispuestas en parcelas individuales de cuatro surcos a $0,7 \mathrm{~m}$ de distancia y de $6 \mathrm{~m}$ de

\footnotetext{
1 Presentado en la XLI Reunión Anual del PCCMCA en Honduras, América Central. 26 de marzo - 1 de abril, 1995.

2 Laboratorio Provincial de Sanidad Vegetal de Holguín. Ministerio de la Agricultura. Cuba.

3 Estación Territorial de Investigaciones Agropecuarias de Holguín. Ministerio de la Agricultura. Cuba.
} 
longitud para un área de $16,8 \mathrm{~m}^{2}$ cada una. El ensayo se repitió al año siguiente.

Las evaluaciones se realizaron a los 60 días de la siembra. Una de ellas consistió en recolectar 20 hojas al azar de los surcos centrales de cada parcela y hacerles un conteo de pústulas, los que se ubicaron dentro de rangos de la siguiente manera:

\section{Grados}

0
1
2
3
4
5

\section{No. De pústulas}

$$
\begin{aligned}
& 0 \\
& 1-10 \\
& 11-30 \\
& 31-60 \\
& 61-100 \\
& \text { más de } 100
\end{aligned}
$$

Esta escala se transformó en grados siguiendo la función $\mathrm{Y}=\div \mathrm{x} / 5$ (Isidrón; Rodríguez; Valkoun, 1979), donde ' $\mathrm{X}$ ' es el número e ' $\mathrm{Y}$ ' el grado de ataque.

Para la interpretación de los datos se utilizó el método no paramétrico de prueba de dos muestras, de Kolmogorov- Smirnov, de una sola cola y muestras pequeñas menores de 40 observaciones con un nivel de significación del 5\%, (Siegel, 1972).

La otra evaluación consistió en medir bajo estereomicroscopio el diámetro de las pústulas para diferenciar el tipo de ataque. La clasificación del grado de susceptibilidad de las variedades se realizó determinando el tamaño promedio del tipo de pústula predominante, según la escala del Vivero Internacional de Roya del Frijol (IBRN) descrita por el CIAT (1984):

\section{Grado Características de las pústulas}

1 Sin evidencias de infección

2 Manchas necróticas sin esporulación

3 Pústulas menor de $0,3 \mathrm{~mm}$

4 Pústulas de 0,3-0,5 $\mathrm{mm}$ y algunas veces rodeadas por halos cloróticos

5 Pústulas mayor de $0,5 \mathrm{~mm}$ y generalmente rodeadas por halos cloróticos

Las variedades de frijol estudiadas, así como los valores promedio de las evaluaciones realizadas en los dos años se presentan en el Cuadro 1.

\section{Segundo experimento}

En la misma área experimental se efectuó otro ensayo durante dos años, con otras 30 variedades de frijol, utilizando un diseño de Bloques al Azar con cuatro repeticiones. Las parcelas fueron de $4 \mathrm{~m}$ de largo y un ancho de cuatro surcos para un área de 11,2 $\mathrm{m}^{2}$.

El porcentaje de grado de ataque se determinó mediante la evaluación de 20 plantas al azar, ubicadas en los surcos centrales de cada parcela con base a una escala de siete grados, de la fórmula de Towsend y Heuberger (Ciba-Geigy, 1981).

La escala utilizada fue la siguiente:

Grados
0
0,1
1
2
3
4
5

\section{Descripción}
Planta sana Aparición de las primeras pústulas Hasta un $20 \%$ del área foliar afectada De 21-40\% del área foliar afectada De $41-60 \%$ del área foliar afectada De $61-80 \%$ del área foliar afectada Más del $80 \%$ del área foliar afectada

Otra evaluación del grado de ataque consistió en realizar un conteo de pústulas por hoja, para lo cual se recolectaron 20 hojas al azar de los surcos centrales de cada parcela. Los datos obtenidos por ambos métodos se procesaron estadísticamente mediante análisis de varianza y la significación entre las variedades se obtuvo por la prueba de Rangos Múltiples de Duncan al $5 \%$. Los valores correspondientes al porcentaje del área foliar afectada fueron previamente transformado a $\mathrm{x}=2$ arc sen $\div$ p.

El tipo de ataque y la clasificación de los cultivares se realizó de forma similar al experimento anterior. En el Cuadro 2, se presentan las variedades estudiadas, así como los valores promedios de las evaluaciones realizadas en los dos años.

\section{RESULTADOS Y DISCUSION}

Las variedades más susceptibles resultaron las comprendidas desde ICTA-Quetzal hasta ICTATamazulapa (Cuadro 1) que arrojaron un número superior a 30 pústulas por hoja, de medianas a grandes y con predominio de halo clorótico. Las comprendidas desde BAT-1280 hasta Güira-35 mostraron resistencia al ataque de la roya con un promedio igual o inferior a 10 pústulas por hoja en general pequeñas y sin clorosis.

Los resultados obtenidos con la variedadd Porrillo sintético coinciden con los de Gálvez; Galindo; Alvarez (1977). Velasco Largo tuvo una respuesta más susceptible con relación a la obtenida por Isidrón et al. (1979), aunque se corresponde con la de González y Castellanos (1983). 
Cuadro 1. Grado de ataque por roya, diámetro de las pústulas predominante y su clasificación, por variedades.

\begin{tabular}{|c|c|c|c|c|c|}
\hline Número & Variedades & Pústula/Hoja & $\begin{array}{l}\text { Grado de } \\
\text { ataque }\end{array}$ & $\begin{array}{l}\text { Diámetro(mm) } \\
\text { de Pústula } \\
\text { Predominante }\end{array}$ & $\begin{array}{l}\text { Clasificación } \\
\text { IBRN } \\
\text { CIAT, 1984) }\end{array}$ \\
\hline 1 & ICTA- Quetzal & $105 \mathrm{a}$ & 4,6 & $0,48(\mathrm{M}+)$ & 4 \\
\hline 2 & ICTA-Jutiapan & $90 \mathrm{~b}$ & 4,2 & $0,63(\mathrm{G}+)$ & 5 \\
\hline 3 & Velasco Largo & $83 \mathrm{~b}$ & 4,1 & $0,68(\mathrm{G}+)$ & 5 \\
\hline 4 & APN- 6 & $82 \mathrm{~b}$ & 4,0 & $0,63(\mathrm{G}+)$ & 5 \\
\hline 5 & BAT- 304 & $70 \mathrm{~b}$ & 3,7 & $0,48(\mathrm{M}+)$ & 4 \\
\hline 6 & DOR- 41 & $69 \mathrm{~b}$ & 3,7 & $0,47(\mathrm{M}+)$ & 4 \\
\hline 7 & Montcalm & $63 \mathrm{~b}$ & 3,5 & $0,34(\mathrm{M}-)$ & 4 \\
\hline 8 & EMP- 84 & $61 \mathrm{~b}$ & 3,5 & $0,42(\mathrm{M}+)$ & 4 \\
\hline 9 & MCD- 253 & $57 \mathrm{bc}$ & 3,3 & 0,40 (M-) & 4 \\
\hline 10 & A- 336 & $51 \mathrm{~cd}$ & 3,1 & $0,34(\mathrm{M}-)$ & 4 \\
\hline 11 & Red Kloud & $49 \mathrm{~d}$ & 3,2 & $0,64(\mathrm{G}+)$ & 5 \\
\hline 12 & Chorotega & $43 \mathrm{~d}$ & 2,9 & $0,36(\mathrm{M}+)$ & 4 \\
\hline 13 & BAT- 24 & $42 \mathrm{~d}$ & 2,9 & $0,61(\mathrm{G}+)$ & 5 \\
\hline 14 & Negro Huasteco-81 & $41 \mathrm{~d}$ & 2,8 & $0,33(\mathrm{M}-)$ & 4 \\
\hline 15 & BAT- 1257 & $40 \mathrm{~d}$ & 2,8 & 0,31 (M-) & 4 \\
\hline 16 & BAT- 832 & $34 \mathrm{~d}$ & 2,6 & $0,36(\mathrm{M}+)$ & 4 \\
\hline 17 & ICTA- Tamazulapa & $32 \mathrm{~d}$ & 2,5 & $0,34(\mathrm{M}+)$ & 4 \\
\hline 18 & Porrillo Sintético & $26 \mathrm{e}$ & 2,3 & $0,37(\mathrm{M}+)$ & 4 \\
\hline 19 & BAT- 450 & 17 ef & 1,8 & $0,49(\mathrm{M}+)$ & 4 \\
\hline 20 & BAT- 68 & $15 \mathrm{ef}$ & 1,7 & $0,32(\mathrm{M}+)$ & 4 \\
\hline 21 & BAT- 448 & $13 \mathrm{fg}$ & 1,6 & $0,20(\mathrm{P}+)$ & 3 \\
\hline 22 & BAT- 518 & $12 \mathrm{~g}$ & 1,5 & $0,42(\mathrm{M}+)$ & 4 \\
\hline 23 & BAT- 1280 & $10 \mathrm{gh}$ & 1,4 & $0,22(\mathrm{P}-)$ & 3 \\
\hline 24 & BAT- 1274 & $9 \mathrm{gh}$ & 1,3 & $0,21(\mathrm{P}-)$ & 3 \\
\hline 25 & Bolita- 42 & $8 \mathrm{gh}$ & 1,2 & $0,35(\mathrm{M}+)$ & 4 \\
\hline 26 & BAT- 1281 & $7 \mathrm{gh}$ & 1,2 & $0,31(\mathrm{M}+)$ & 4 \\
\hline 27 & Huetar & $7 \mathrm{gh}$ & 1,2 & $0,15(\mathrm{P}-)$ & 3 \\
\hline 28 & Tazumal & $7 \mathrm{gh}$ & 1,2 & $0,32(\mathrm{M}+)$ & 4 \\
\hline 29 & BAT- 202 & $6 \mathrm{~h}$ & 1,1 & $0,18(\mathrm{P}-)$ & 3 \\
\hline 30 & Rosas & $3 \mathrm{i}$ & 0,8 & $0,18(\mathrm{P}-)$ & 3 \\
\hline 31 & M- 112 & $1 \mathrm{j}$ & 0,4 & $0,17(\mathrm{P}-)$ & 3 \\
\hline 32 & Revolución- 79 & $0 \mathrm{i}$ & 0,0 & Manchas Necróticas & 2 \\
\hline 33 & XAN- 43 & $0 \mathrm{j}$ & 0,0 & Manchas Necróticas & 2 \\
\hline 34 & G_ira- 35 & $0 \mathrm{j}$ & 0,0 & Manchas Necróticas & 2 \\
\hline
\end{tabular}

Letras distintas indican diferencias significativas al 5\% (Kolmogorov- Smirnov).

$\mathrm{P}=$ Pústulas pequeñas (menor de $0,3 \mathrm{~mm}$ ) + con halo clorótico

$\mathrm{M}=$ Pústulas medianas ( de 0,3 a $0,5 \mathrm{~mm}$ ) - Sin halo clorótico

$\mathrm{G}=$ Pústulas grandes (mayor de $0,5 \mathrm{~mm}$ ) 
Cuadro 2. Grado de ataque por roya, diámetro de las pústulas predominantes y su clasificación, por variedades.

\begin{tabular}{|c|c|c|c|c|c|}
\hline \multirow{2}{*}{$\begin{array}{c}\text { Número } \\
1\end{array}$} & \multirow{2}{*}{$\begin{array}{l}\text { Variedades } \\
\text { ICA- Pijao }\end{array}$} & \multicolumn{2}{|c|}{$\begin{array}{c}\text { Grado de ataque } \\
\text { Pústulas/hoja } \\
(\%)\end{array}$} & \multirow{2}{*}{$\begin{array}{c}\begin{array}{c}\text { Diámetro }(\mathbf{m m}) \\
\text { de la pústula } \\
\text { Predominante }\end{array} \\
0,90(\mathrm{G}+)\end{array}$} & \multirow{2}{*}{$\begin{array}{c}\begin{array}{c}\text { Clasificación } \\
\text { IBRN } \\
\text { (CIAT, 1984) }\end{array} \\
5\end{array}$} \\
\hline & & $85 \mathrm{a}$ & $97 \mathrm{a}$ & & \\
\hline 2 & Güira- 89 & $81 \mathrm{a}$ & $90 \mathrm{a}$ & $0,80(\mathrm{G}+)$ & 5 \\
\hline 3 & 78-03-74 & $75 \mathrm{a}$ & $88 \mathrm{a}$ & $0,64(\mathrm{G}+)$ & 5 \\
\hline 4 & PUBZ- 1881 & $69 \mathrm{ab}$ & $84 \mathrm{a}$ & $0,38(\mathrm{M}+)$ & 4 \\
\hline 5 & IPA- 7419 & $53 \mathrm{bc}$ & $60 \mathrm{~b}$ & $0,21(\mathrm{P}-)$ & 3 \\
\hline 6 & A- 231 & $48 \mathrm{~cd}$ & $58 \mathrm{~b}$ & $0,43(\mathrm{M}+)$ & 4 \\
\hline 7 & Hatuey 24 & $46 \mathrm{~cd}$ & $44 \mathrm{c}$ & $0,74(\mathrm{G}+)$ & 5 \\
\hline 8 & Bonita 11 & 43 cde & $52 \mathrm{~b}$ & $0,66(\mathrm{G}+)$ & 5 \\
\hline 9 & ICTA 81-64 & 39 cde & $34 \mathrm{~cd}$ & $0,43(\mathrm{M}+)$ & 4 \\
\hline 10 & Corobici & $35 \mathrm{de}$ & $33 \mathrm{~cd}$ & $0,72(\mathrm{G}+)$ & 5 \\
\hline 11 & ICTA- Ostua & $27 \mathrm{e}$ & 30 cde & $0,40(\mathrm{M}+)$ & 4 \\
\hline 12 & CC 25-9 (B) & $26 \mathrm{e}$ & $32 \mathrm{cde}$ & $0,19(\mathrm{P}-)$ & 3 \\
\hline 13 & XAN 68 & $17 \mathrm{f}$ & 27 cdef & $0,45(\mathrm{M}+)$ & 4 \\
\hline 14 & CC 25-9 (N) & $12 \mathrm{fg}$ & 18 efg & $0,40(\mathrm{M}+)$ & 4 \\
\hline 15 & NAG 15 & $10 \mathrm{fgh}$ & 20 defg & $0,69(\mathrm{G}+)$ & 5 \\
\hline 16 & CC 25-9-T (R) & $9 \mathrm{fgh}$ & 19 defg & $0,37(\mathrm{M}+)$ & 4 \\
\hline 17 & BAT 1198 & $8 \mathrm{fgh}$ & 15 fghi & 0,19 (P-) & 3 \\
\hline 18 & DOR 224 & 6 ghi & 13 fghi & $0,20(\mathrm{P}-)$ & 3 \\
\hline 19 & CC 25-9-S (R) & $5 \mathrm{hij}$ & 9 ghi & $0,30(\mathrm{M}+)$ & 4 \\
\hline 20 & RAB 30 & 5 hij & 10 ghi & $0,14(\mathrm{P}-)$ & 3 \\
\hline 21 & NAG 55 & 4 hij & 7 ghi & $0,21(\mathrm{P}-)$ & 3 \\
\hline 22 & NAG 20 & 3 hij & 9 ghi & $0,21(\mathrm{P}-)$ & 3 \\
\hline 23 & XAN 147 & $2 \mathrm{ijk}$ & $2 \mathrm{hi}$ & $0,15(\mathrm{P}-)$ & 3 \\
\hline 24 & XAN 93 & $2 \mathrm{ijk}$ & $2 \mathrm{hi}$ & $0,20(\mathrm{P}-)$ & 3 \\
\hline 25 & Chévere & $1 \mathrm{jk}$ & $2 \mathrm{hi}$ & $0,16(\mathrm{P}-)$ & 3 \\
\hline 26 & MCD 254 & $0 \mathrm{k}$ & $1 \mathrm{i}$ & $0,18(\mathrm{P}-)$ & 3 \\
\hline 27 & Guamá 23 & $0 \mathrm{k}$ & $1 \mathrm{i}$ & $0,16(\mathrm{P}-)$ & 3 \\
\hline 28 & Engañador & $0 \mathrm{k}$ & $0 \mathrm{i}$ & Manchas Nec & Sticas 2 \\
\hline 29 & Bat- 1275 & $0 \mathrm{k}$ & $0 \mathrm{i}$ & Manchas Nec & Sticas 2 \\
\hline 30 & PV 555 & $0 \mathrm{k}$ & $0 \mathrm{I}$ & Manchas Nec & Sticas 2 \\
\hline
\end{tabular}

Sx: $0,09 \quad 4,42$

Cv: $16,98 \quad 27,64$

Letras distintas indican diferencias significativas al 5\% (TRM- Duncan)

$\mathrm{P}=$ Pústulas pequeñas (menor de $0,3 \mathrm{~mm}$ ) + con halo clorótico

$\mathrm{M}=$ Pústulas medianas ( de 0,3 a $0,5 \mathrm{~mm}$ ) - sin halo clorótico

$\mathrm{G}=$ Pústulas grandes ( mayor de $0,5 \mathrm{~mm}$ ) 
El comportamiento de ICTA-Quetzal, BAT-518 y Tazumal resultó similar al referido por Gálvez; Orozco; Voysest (1985). En cambio con las variedades EMP-84 e ICTA-Tamazulapa que según Pastor-Corrales (1985) resultaron resistentes en la provincia de La Habana, en el presente estudio arrojaron un comportamiento de susceptible, lo que podría deberse a la existencia de diferentes razas de roya en una u otra localidad.

En el Cuadro 2 se observa que los cultivares más severamente atacados, desde ICA- Pijao hasta CC 25-9 (B), tuvieron un valor superior al $25 \%$ y a 30 pústulas por hoja respectivamente, como grado de ataque; presentaron pústulas de medianas a grandes con predominio de halo clorótico y resultaron susceptibles según la clasificación utilizada. Lo contrario ocurrió con las variedades comprendidas desde CC 25-9-S (R) hasta PV-555, que arrojaron un promedio igual o inferior a 10 pústulas por hoja (de 0 a $5 \%$ de grado de ataque) y mostraron resistencia al ataque de la enfermedad.

El comportamiento de las variedades ICA- Pijao, Bonita 11 y CC 25-9-T (R) se corresponde con los obtenidos por Quintero et al. (1988). Las variedades Chévere y Engañador tuvieron una respuesta de mayor resistencia con relación a la obtenida por dichos autores en la región central de Cuba.

\section{LITERATURA CITADA}

CENTRO INTERNACIONAL DE AGRICULTURA TROPICAL (CIAT). 1984. Vivero internacional de roya del frijol (IBRN). Resultados 1979-1980. Cali. Colombia p. 4-5.
CIBA-GEIGY. 1981. Manual para ensayos de campo en protección vegetal. 2 da Edición. Basilia. Suiza. p.34.

GALVEZ, G. ; GALINDO, J.; ALVAREZ, G. 1977. Defoliación artificial para estimar pérdidas por daños foliares en frijol (Phaseolus vulgaris L.). Turrialba 27 (2): 143-146.

GALVEZ, G.; OROZCO, S.; VOYSEST, O. 1985. Comentarios relacionados con el problema de la roya del frijol en Cuba. CIAT. Cali. Colombia. 5 p.

GOZALEZ, M.; CASTELLANOS, J. 1983. Razas fisiológicas de la roya del frijol (Uromyces phaseoli var. Typica) en Cuba. Ciencias de la Agricultura 15: 3-5.

GONZALEZ, M. 1984. Enfermedades fungosas del frijol en Cuba. Conferencia. Curso de Postgrado. Instituto de Investigaciones de Sanidad Vegetal. Ciudad de La Habana. Cuba. P. 2-8.

ISIDRON, M.; RODRIGUEZ, J.; VALKOUN, J. 1979. Evaluación de la resistencia a la roya Uromyces phaseoli de 15 variedades de frijol en condiciones de campo. Agrotecnia de Cuba 11 (1): 115-122.

PASTOR-CORRALES, M. 1985. el uso de mezclas de variedades para el control o manejo de enfermedades. CIAT. Cali. Colombia. 7 p.

QUINTERO,E.; CARAZA, R.; ABREU, O.; HERNÁNDEZ,A. 1988. Comportamiento de veinte variedades de frijol común (Phaseolus vulgaris L.) en la Región Central de Cuba. Centro Agrícola XV (2):16-23.

SIEGEL, S. 1972. Diseño experimental no paramétrico. E. R. Instituto del Libro. La Habana. P. 155-165. 